\title{
Bunch-by-bunch linac beam energy profile diagnostics using storage ring phase rotation and synchrotron radiation
}

\author{
Takahiro Matsubara, Shintarou Hisao, and Yoshihiko Shoji \\ Laboratory of Advanced Science and Technology for Industry (LASTI), University of Hyogo, \\ 1-1-2 Kouto, Kamigori-cho, Ako-gun, Hyogo 678-1205, Japan \\ Takao Asaka and Shinsuke Suzuki \\ Japan Synchrotron Radiation Research Institute (JASRI), SPring-8, 1-1-1 Kouto, Sayo-cho, Sayo-gun, Hyogo 679-5198, Japan
}

(Received 19 May 2005; published 4 April 2006)

\begin{abstract}
This paper reports a new method of measuring the bunch-by-bunch energy profile of a linear accelerator (linac) electron beam, using a dual-sweep streak camera in the storage ring. This method records the evolution of the time profile in the storage ring following injection. A high-resolution linac beam energy profile is reconstructed from the time profile measured at one-quarter of a synchrotron oscillation period following injection. Conversion between the time profile and energy profile is based on results from a longitudinal phase space tracking simulation. By separating the streak camera images of the different bunches, a bunch-by-bunch energy profile can also be measured.
\end{abstract}

DOI: $10.1103 /$ PhysRevSTAB.9.042801

PACS numbers: 41.85.Ew, 29.17.+w, 29.30.Aj, 41.85.Qg

\section{INTRODUCTION}

Recent progress in the performance of electron linear accelerators (linac) has increased the demand for better beam diagnostics to improve performance in daily operations. In a synchrotron radiation storage ring, for instance, accurate information on the linac beam used for top-up injection is absolutely necessary because beam excursions in both the transverse and longitudinal directions must be minimized. New linac beam diagnostics will also improve our knowledge of beam dynamics, especially in the context of fast, bunch-by-bunch diagnostics since information on beam loading and the bunching process is provided.

Several linac diagnostics currently exist to measure the beam energy profile. The most common one uses a screen monitor or wire scanner in a dispersion section of the beam transport line. Although this method is both easy and useful, it has poor time resolution and the contribution from transverse oscillation must be corrected for. The observation of optical transition radiation (OTR) using a fast-gated CCD camera [1] has sufficient time resolution for bunch-by-bunch measurements. The OTR is not bright enough, however, for a single-shot measurement [2]. The third method, a multi-strip-line energy spread monitor [3], can provide the bunch-by-bunch energy spread of a single shot but does not provide the structure of the energy distribution. A fourth method is to measure the bunch time profile after a $90^{\circ}$ rotation in the longitudinal phase space, using off-crest rf acceleration and a magnetic chicane [4].

Here a new method is described that uses the storage ring as a phase rotator, and a dual-sweep streak camera to detect the time profile evolution. A high-resolution energy profile is reconstructed from the time profile after a $90^{\circ}$ rotation in the rf bucket (one-quarter of a synchrotron oscillation period) following injection. The method is sufficient for a single-shot measurement, since the synchrotron light from several revolutions is accumulated in the camera at a $90^{\circ}$ rotation. Since the linac bunch lengths are very short, the beam occupancy resembles a short, straight line in the rf bucket of the storage ring. A complicated tomographic analysis is therefore unnecessary $[5,6]$. The transverse oscillation contribution is negligible, since only the longitudinal phase space profile is used. Furthermore, bunch-by-bunch energy profiles can be obtained through separate measurements of the different bunches.

The time profile evolution following injection has been measured in proton synchrotrons and more recently in synchrotron radiation rings $[7,8]$. A time profile includes information such as the longitudinal dipole mismatch, quadrupole bucket mismatch, and nonlinear synchrotron tune. This information has been used in beam tuning and the measurement of storage ring parameters. The present article shows that high-resolution time profile diagnostics can be obtained not only for the injected beam in the storage ring, but also for the linac beam itself. This method is very useful in understanding the origins of beam loss in a top-up storage ring injection process, and provides reliable information on beam loading in the linac.

Section II introduces the analytical formulas describing the movement of an electron in an rf bucket. Section III demonstrates the method by measuring the bunch-bybunch energy profiles of the SPring-8 linac [9-11] using a streak camera in the NewSUBARU storage ring [12]. A single-bunch measurement is presented initially, along with an explanation of the nonlinear correction method. Next, the techniques used to separately measure different bunches (bunch-by-bunch measurement) are explained. Section IV discusses the energy resolution of the measurement and possible sources of error. Finally, Appendices A 
and B explain some small effects on the measurement that can be safely neglected.

\section{ANALYTICAL FORMULAS FOR BUNCH ROTATION IN AN RF BUCKET}

This method is based on the well-known theory of bunch rotation in an $\mathrm{rf}$ bucket (in the longitudinal phase space). Equations (1a) and (1b) are linear approximations to the equations of synchrotron oscillation:

$$
\begin{aligned}
& d \tau=\alpha_{1} T_{\mathrm{REV}} \delta, \\
& d \delta=-\frac{\omega_{S}^{2} T_{\mathrm{REV}}}{\alpha_{1}} \tau .
\end{aligned}
$$

Here $\delta$ and $\tau$ are the relative energy displacement and time displacement of the reference particle, respectively, while $d \delta$ and $d \tau$ are the changes in these quantities per revolution. $\alpha_{1}$ is the linear momentum compaction factor, $T_{\mathrm{REV}}$ is the revolution period, and $\omega_{S}$ is the synchrotron oscillation angular frequency given by

$$
\omega_{S}^{2}=-\frac{e V_{\mathrm{rf}} \omega_{\mathrm{rf}} \alpha_{1} \cos \phi_{S}}{E_{0} T_{\mathrm{REV}}} .
$$

In Eq. (2), $V_{\mathrm{rf}}$ is the rf acceleration voltage, $\omega_{\mathrm{rf}}$ is the $\mathrm{rf}$ angular frequency, $\phi_{s}$ is the synchronous phase, and $E_{0}$ is the reference energy.

Suppose that an electron is injected at point $\left(\tau_{0}, \delta_{0}\right) . \tau_{1}$ is defined as the time displacement after a $90^{\circ}$ rotation, so that $\tau_{1}$ is independent of $\tau_{0}$. The solution to Eqs. (1a) and (1b) is

$$
\tau_{1}=\frac{\alpha_{1}}{\omega_{S}} \delta_{0}
$$

Accordingly, the initial energy displacement $\delta_{0}$ is obtained from $\tau_{1}$ using Eq. (3).

In a real machine where the rf bucket is not linear, $\tau$ is expressed as a function of time and the initial state, $\tau=$ $f\left(\delta_{0}, \tau_{0}, t\right)$, where $t$ is the time since injection.

Since the occupancy of the linac beam in the rf bucket can be approximated by a short, straight line, $\tau_{1}$ is the function $f\left(\delta_{0}, 0, T_{\text {MEAS }}\right) \equiv f_{0}\left(\delta_{0}\right) . \tau_{1}$ is obtained by tracking test particles using the given parameters. $T_{\text {MEAS }}$ is the measurement time, which is close to $1 / 4$ of the synchrotron oscillation period.

A phase rotation transfers the initial energy profile $D\left(\delta_{0}\right)$ to the time profile $T\left(\tau_{1}\right)$ measured by the streak camera. The time displacement $\tau_{1}$ is converted into the initial energy $\delta_{0}$ using

$$
\delta_{0}=f_{0}^{-1}\left(\tau_{1}\right) .
$$

The conversion of the density function $T\left(\tau_{1}\right)$ to $D\left(\delta_{0}\right)$ can be obtained by

$$
D\left(\delta_{0}\right)=\left(d f_{0} / d \delta_{0}\right) T\left(\tau_{1}\right),
$$

since they satisfy the relation

$$
\int_{-\infty}^{\delta_{0}} D(\delta) d \delta=\int_{-\infty}^{\tau_{1}} T(\tau) d \tau .
$$

\section{MEASUREMENT}

\section{A. Measurement setup}

Figure 1 shows the layout of the SPring-8 linac, the $8 \mathrm{GeV}$ booster synchrotron, and the NewSUBARU storage ring. Tables I and II show the main parameters of the linac

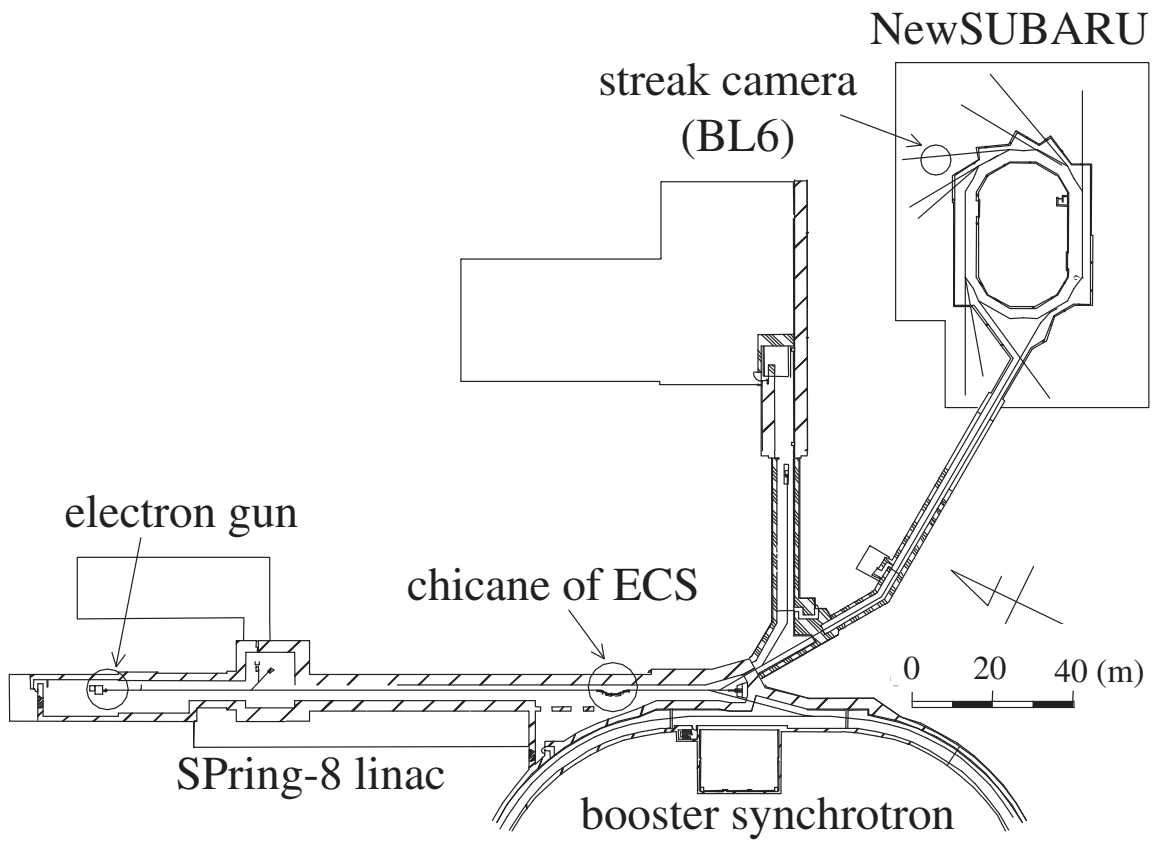

FIG. 1. Layout of the SPring-8 linac, booster synchrotron, and NewSUBARU storage ring. 
TABLE 1. Main parameters of the NewSUBARU storage ring.

\begin{tabular}{lc}
\hline \hline \multicolumn{1}{c}{ Parameters (at $1 \mathrm{GeV})$} & \\
\hline rf frequency $\left(\omega_{\text {rf }} / 2 \pi\right)$ & $499.955 \mathrm{MHz}$ \\
Synchrotron oscillation frequency $\left(\omega_{S} / 2 \pi\right)$ & $6 \mathrm{kHz}$ at $V_{\text {rf }}=130 \mathrm{kV}$ \\
Synchrotron oscillation damping time $\left(\tau_{\varepsilon}\right)$ & $10 \mathrm{kHz}$ at $V_{\text {rf }}=400 \mathrm{kV}$ \\
Harmonic number & $12 \mathrm{~ms}$ \\
Linear momentum compaction factor $\left(\alpha_{1}\right)$ & 198 \\
Natural energy spread $\left(\delta_{N}\right)$ & 0.0013 \\
\hline \hline
\end{tabular}

TABLE 2. Main parameters of the SPring-8 linac .

\begin{tabular}{lcc}
\hline \hline Parameters (at 1 GeV) & Without ECS & With ECS \\
\hline rf frequency & \multicolumn{2}{c}{$2856 \mathrm{MHz}$} \\
rf period & \multicolumn{2}{c}{$350 \mathrm{ps}$} \\
Transverse emittance & $\sim 71 \pi \mathrm{nmrad}$ at $1 \mathrm{GeV}$ \\
Micro bunch charge & $0.03 \sim 0.3 \mathrm{nC}$ \\
Micro bunch length (FWHM) & $10 \mathrm{ps}$ & $20 \mathrm{ps}$ \\
Energy spread (full width) & $1.0 \%$ & $0.60 \%$ \\
Energy stability (rms) & $0.06 \%$ & $0.01 \%$ \\
\hline \hline
\end{tabular}

and the storage ring. The linac supplies a $1 \mathrm{GeV}$ electron beam to the synchrotron and the storage ring. The gridcathode voltage pulse width of the electron gun determines the macropulse width of the linac beam. A pulse width of $0.25 \mathrm{~ns}$ was used in these experiments for a single microbunch, and $1 \mathrm{~ns}$ for three or four bunches. An energy compression system (ECS) $[13,14]$ was placed at the end of the linac to stabilize the beam energy and to reduce the energy spread.

Figure 2 depicts the layout of the optical equipment from the light source to the streak camera (C6860 SYNCHRO FESCA, Hamamatsu Photonics) in the bending magnet beam line (BL6). The white radiation beam from the magnet was introduced to the camera using a pair of focusing mirrors. The width of the entrance slit in the first mirror determined the acceptance of the line. Four jaw slits were placed in a line upstream of the flat insert mirror to reject unwanted light. The camera was operated in doublesweep mode with a fast-sweep frequency of $83.3 \mathrm{MHz}, 1 / 6$ of the rf clock signal, and was triggered by the injection timing signal with a variable delay.

\section{B. Measurement of a single-bunch beam}

A single-bunch beam with a pulse width of $0.25 \mathrm{~ns}$ was measured to determine the characteristics of this method. The rf phase of the storage ring was adjusted so that the bunch was injected at a point near the center of the rf bucket. A composite image of 50 injections is used in this experiment to improve clarity. Figure 3(a) is a streak camera image of the beam transient following the injection. The vertical (fast-sweep) axis shows the bunch length, while the horizontal (slow-sweep) axis shows the evolution of the profile. The camera observed synchrotron light for $100 \mu$ s along the slow-sweep axis, which was about half of the synchrotron oscillation period. The full scale of the fast-sweep axis was $500 \mathrm{ps,} \mathrm{which} \mathrm{was} \mathrm{a} \mathrm{quarter} \mathrm{of} \mathrm{the}$ storage ring's rf period. Figure 3(b) shows the intensity profile $T\left(\tau_{1}\right)$ at $T_{\text {MEAS }}$. The slice width along the slowsweep axis is $1.9 \mu \mathrm{s}$ (5 revolutions).

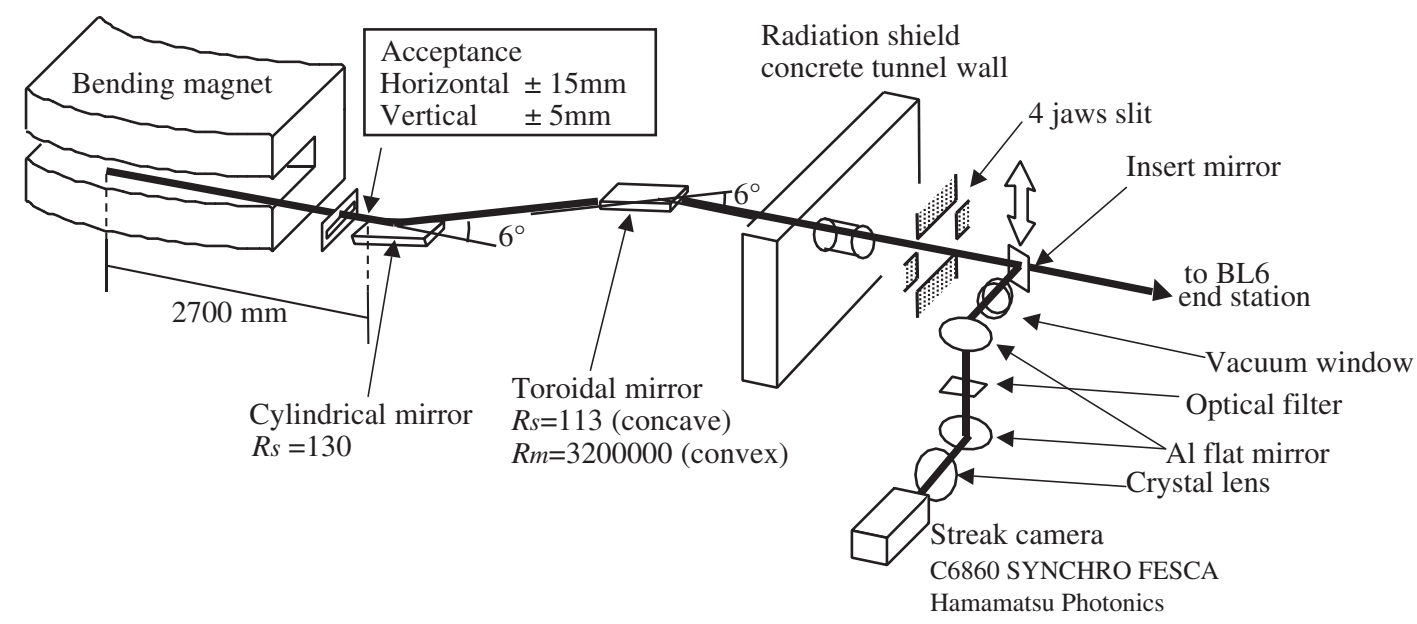

FIG. 2. Layout of the BL6 mirrors and optical equipment before the streak camera. 


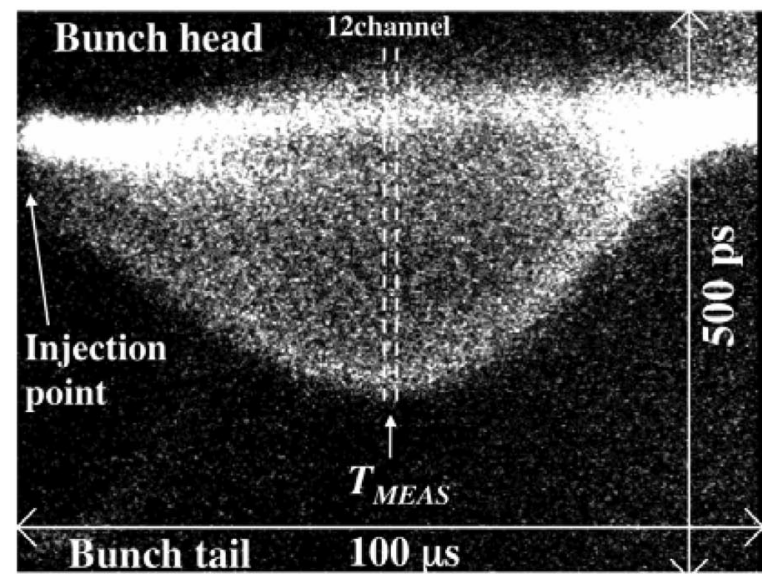

(a)

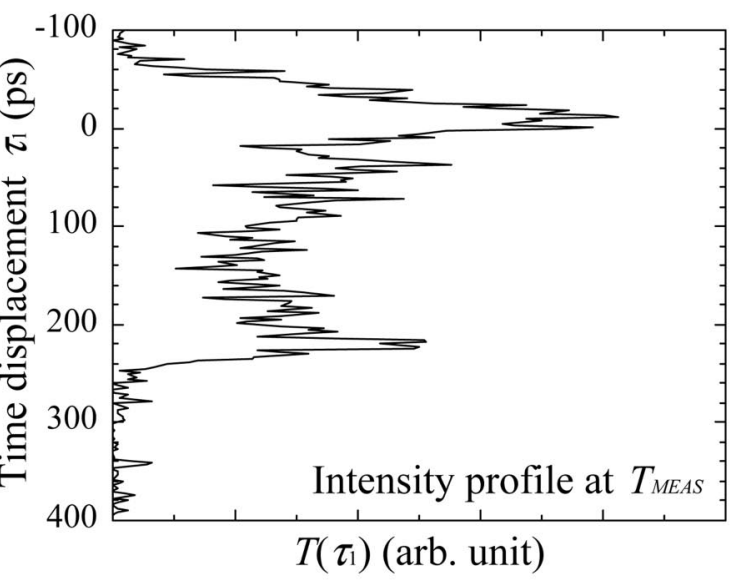

(b)

FIG. 3. Streak camera image (accumulation of 50 shots). (a) shows the longitudinal transient of the injected beam, and (b) shows the intensity profile $T\left(\tau_{1}\right)$ at $T_{\text {MEAs }}$. The pulse width is $0.25 \mathrm{~ns}$ and the rf voltage is $130 \mathrm{kV}$. The bunch charge is approximately $0.03 \mathrm{nC}$. The broken lines in (a) indicate the slice width (about $1.9 \mu \mathrm{s}$ ) for (b).

The time profile of Fig. 3(b) corresponds to a rough energy profile, with the conversion between the time axis and energy axis given by Eq. (3). An accurate energy profile $D\left(\delta_{0}\right)$ was also reconstructed from $T\left(\tau_{1}\right)$, using Eqs. (4) and (5). The linear and nonlinear momentum compaction factors were obtained through independent measurements using the stored beam [15]. These factors can be approximated by

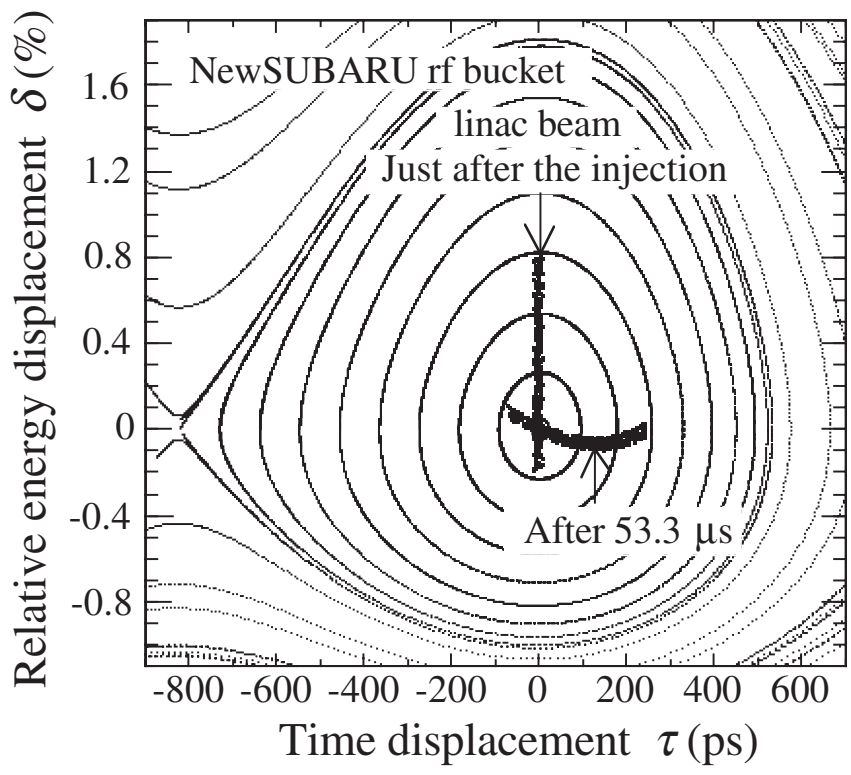

FIG. 4. Result of a particle tracking simulation for the NewSUBARU rf bucket $\left(V_{\mathrm{rf}}=130 \mathrm{kV}\right)$. A uniform rectangular distribution, with a 20 ps bunch length and $1.0 \%$ energy spread, are used for the linac beam. The center energy is $+0.3 \%$. The thick lines represent the beam occupancy at injection $(t=0)$ and measurement $\left(t=T_{\text {MEAS }}=53.3 \mu \mathrm{s}\right)$.

$$
\begin{aligned}
& \alpha_{1} \delta+\alpha_{2} \delta^{2}+\alpha_{3} \delta^{3}+\alpha_{4} \delta^{4}+\ldots \\
& \quad \approx 0.0013 \delta-0.081 \delta^{2}+2.741 \delta^{3}-29.1 \delta^{4},
\end{aligned}
$$

where $\alpha_{n}$ is the $n$th order momentum compaction factor. A 4th order polynomial approximation was sufficient for the full acceptance energy range of the storage ring. Figure 4 shows the result of the rf bucket phase space tracking simulation. Figure 5 shows the reconstructed energy profile $D\left(\delta_{0}\right)$. Section IV will discuss the scaling errors in detail.

\section{Measurement of a multibunch beam}

In this experiment a pulse width of $1 \mathrm{~ns}$, which contained three bunches, was used for injection into NewSUBARU.

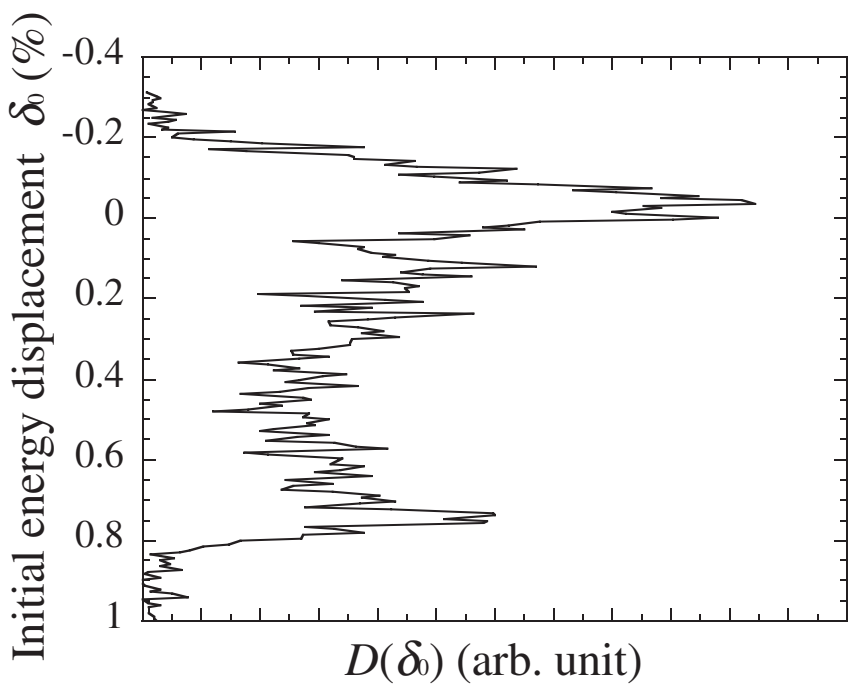

FIG. 5. The energy profile $D\left(\delta_{0}\right)$ of the linac beam, as reconstructed from the time profile $T\left(\tau_{1}\right)$ of Fig. 3(b) using Eqs. (4) and (5). 
The pulse contained three bunches. To obtain the bunchby-bunch profile, it was first necessary to separate the bunch images.

Figures 6(a) and 6(b) are images taken with a normal operating voltage and high storage ring rf voltage, respectively. The three bunches (referred to as \#1, \#2, and \#3) were consecutively injected into the $\mathrm{rf}$ bucket of the synchrotron. A target bunch was selected by adjusting the injection phase. Bunch \#2 was the target of this measurement.

The image of the target bunch overlaps with those of the other bunches at $T_{\text {MEAS }}$, as shown in Fig. 6(a), which prevents a bunch-by-bunch measurement. Applying a higher rf voltage separated the bunches, however, as shown in Fig. 6(b). The higher rf voltage produced a larger energy displacement in the off-timing injected bunches at $T_{\text {MEAS }}$, and separated them through the following processes.

(A) The larger energy displacement increased the radial displacement of the beam center, which made it easier to stop the light from the off-timing bunches. The light from bunch \#3 was stopped at $T_{\text {MEAS }}$ using the four jaw slits set along the beam line, as shown in Fig. 6(b).

(B) The larger energy displacement increased the synchrotron oscillation phase shift of the off-timing injected bunches by the nonlinear momentum compaction factor. This process effectively separated the image of bunch \#1 from that of \#2, as shown in Fig. 6(b).

(C) The higher rf voltage reduced the bunch length at $T_{\text {MEAS }}$ (the spread of $\tau_{1}$ ). This process made it easier to separate the image of bunch \#1 from that of \#2.

Figure 7 shows a simulation of the streak camera image that demonstrates the effects of processes (B) and (C).

\section{Application: The effect of an ECS}

The effect of an energy compression system (ECS) was demonstrated by measuring the bunch-by-bunch energy profile of a pulse with two bunches. Each bunch's total charge was approximately $0.06 \mathrm{nC}$. Figure 8 shows energy profiles measured by the streak camera for 80 injections of the front (\#1) and rear (\#2) bunches. The bunch-by-bunch energy profiles in Fig. 8 clearly show the following characteristics:

(i) The energy profiles changed were narrower in lower rf fields.

(ii) The energy profile of the rear bunch was narrower than that of the front bunch, and this difference grew more pronounced in lower if fields.

(iii) The difference in center energy (halfway between the highest and lowest energies) between the front and rear bunches was larger in lower rf fields.

The total energy spread of the bunches shown in Fig. 8 changed from $0.45 \%$ (corresponding to the width containing $95 \%$ of the profile area) to $0.75 \%$ as the rf field was raised from 3.3 to $9.2 \mathrm{MV} / \mathrm{m}$. This result was consistent with that obtained from a screen monitor, which measured a change from $0.38 \%$ to $0.80 \%$. The screen monitor was set in the linac-NewSUBARU beam transport line at a $2.8 \mathrm{~m}$ dispersion. The betatron oscillation amplitude contributed approximately $\pm 0.07 \%$ to the energy spread.

\section{DISCUSSION}

\section{A. Energy resolution}

The main factor determining the profile energy resolution was the static time resolution of the streak camera $\Delta t_{1}$, which depends on the focused beam size or slit width at the camera aperture. In the measurements described in this article, the static resolution was set to about $1 / 40$ of the full measurement range, corresponding to $\Delta \tau_{1}=13.5 \mathrm{ps}$ (95\% width). The energy resolution calculated by Eq. (3) was found to be $0.039 \%$ (95\% width) in Fig. 5 (the singlebunch measurement) and $0.066 \%$ in Fig. 8 (the multibunch

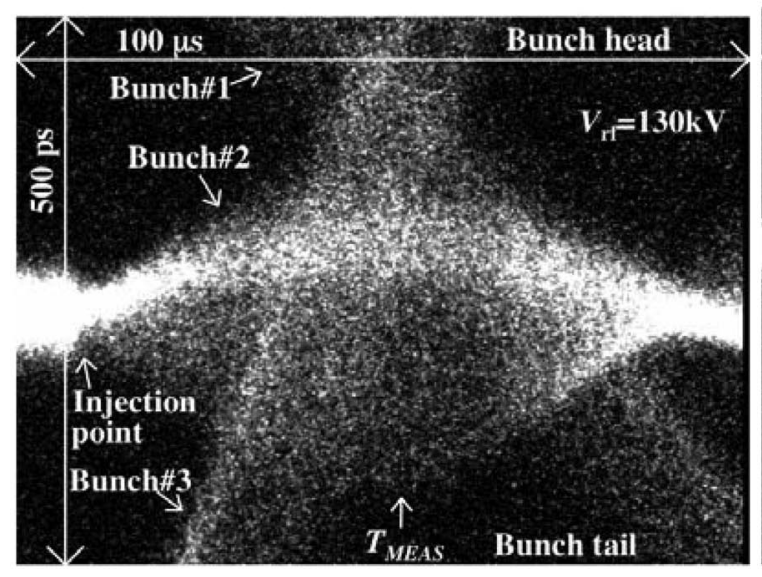

(a)

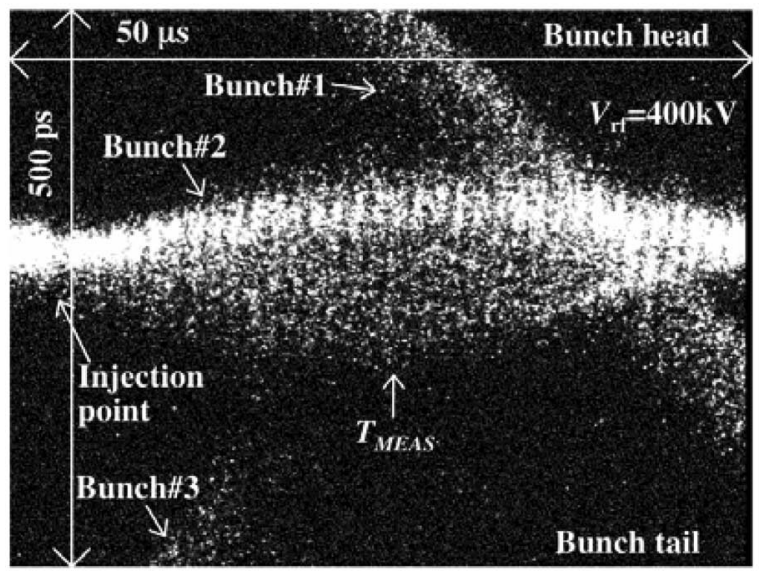

(b)

FIG. 6. Streak camera images with a $1 \mathrm{~ns}$ linac pulse width. (a) uses a normal operation voltage $\left(V_{\mathrm{rf}}=130 \mathrm{kV}\right)$ and (b) uses a high rf voltage $\left(V_{\mathrm{rf}}=400 \mathrm{kV}\right)$. The pulse contains three bunches, \#1, \#2, and \#3, which are consecutively injected into the storage ring. 


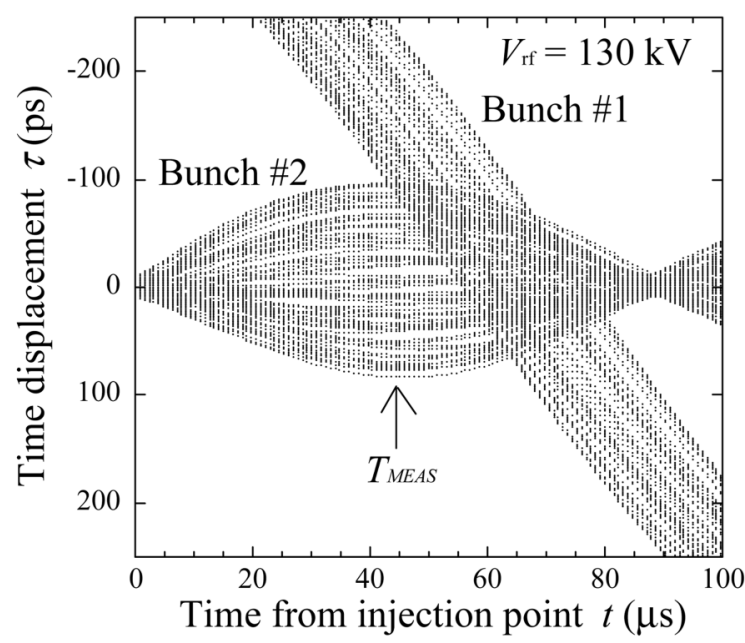

(a)

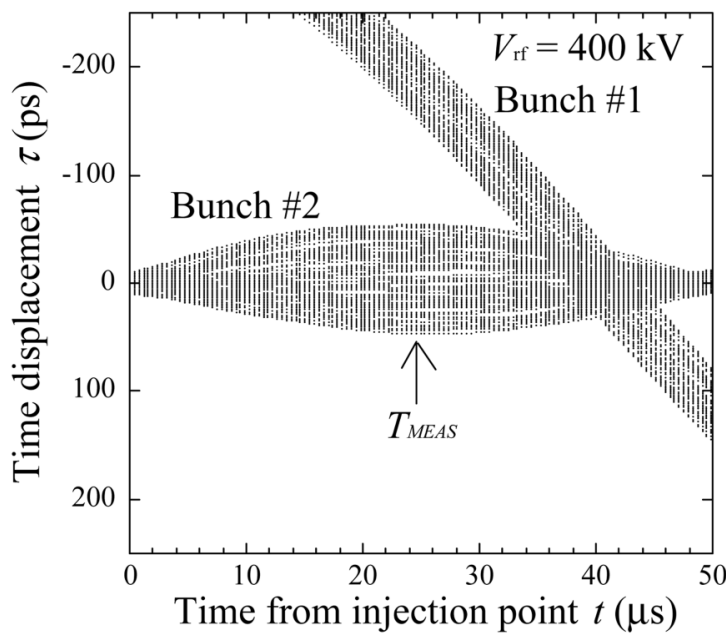

(b)

FIG. 7. Streak camera image of bunches \#1 and \#2, for simulation results with (a) a normal operation voltage and (b) a high rf voltage. The specified beam parameters are $0.5 \%$ for the full energy spread and $20 \mathrm{ps}$ for the bunch length. The image is a trace of 200 particles per bunch.

measurement). In Fig. 8, however, it would have been possible to choose $200 \mathrm{ps}$ as the fast-sweep range rather than 500 ps since the higher $V_{\text {rf }}$ reduced the bunch length at $T_{\text {MEAS }}$. A static resolution of $0.026 \%$ is therefore theoretically possible.

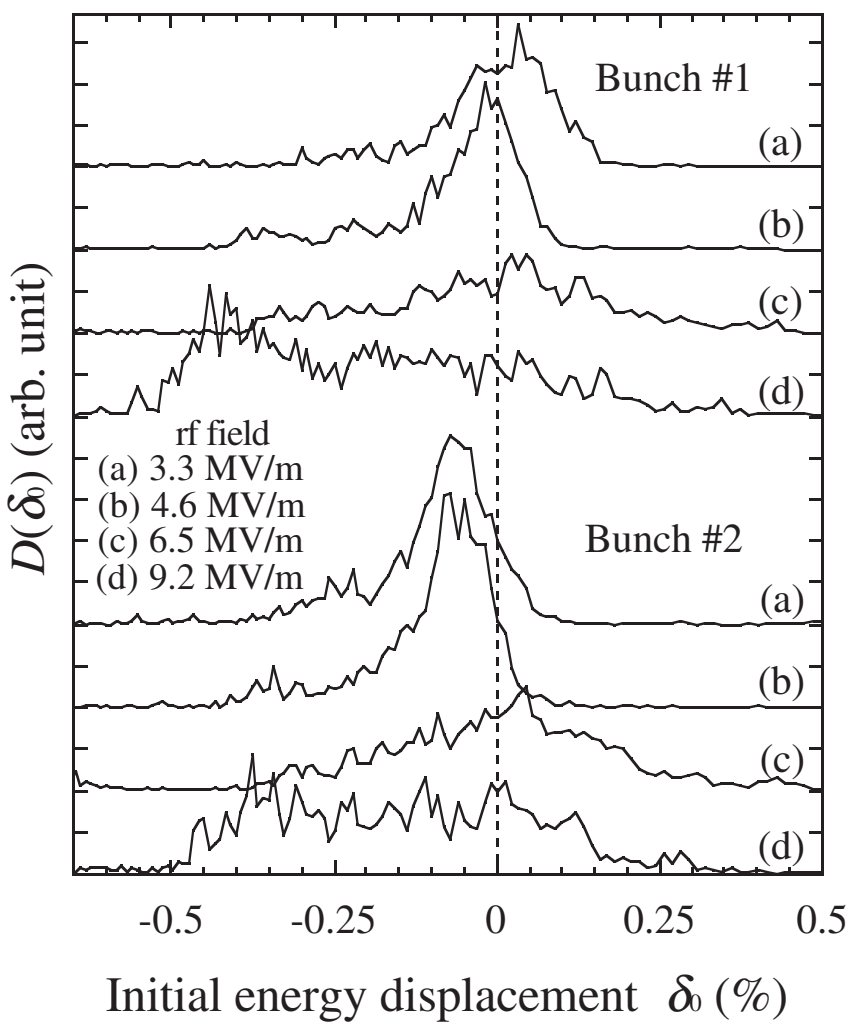

FIG. 8. Energy profiles of the front bunch (\#1) and rear bunch (\#2) for various rf fields, using an ECS.
Other small factors contributing to the energy resolution are explained in Appendixes A and B. Considering these factors, the total resolution has a small dependence on $\delta_{0}$ and its worst value of $0.06 \%$ (95\% width) occurs at $\delta_{0} \approx 0$ in Fig. 5. This decreased resolution was a trade-off, however, for the increased brightness of the measurement.

Another known small factor, not detailed in this paper, is the tiny coupling between transverse motion and longitudinal motion in the storage ring. Its estimated contribution is $0.6 \mathrm{ps}$ to the time spread at BL6 [16], which is negligible as previously mentioned.

\section{B. Scaling errors}

Figure 9 shows the scaling function $f_{0}^{-1}\left(\tau_{1}\right)$ and the density correction function $d f_{0} / d \delta_{0}$, which are used to convert $T\left(\tau_{1}\right)$ to $D\left(\delta_{0}\right)$ in a single-bunch measurement. These functions were obtained through a particle tracking simulation in the rf bucket, as shown in Fig. 4. The corrections determined by the simulation were less than $+8.5 \%$ for the $\delta_{0}$ scale and $-18 \%$ for the density. When a bunch was injected into the center of the rf bucket by adjusting the reference energy of the storage ring, however, these corrections dropped to $+5.6 \%$ for the $\delta_{0}$ scale and $-14.5 \%$ for the density.

In the case of higher rf voltages for the multibunch measurement, nonlinear effects were enlarged on the offtiming injected bunch center but not on the target bunch.

\section{Single-shot measurement}

A single-shot image is sufficient for a rough energy profile. Figures 10(a) and 10(b) show energy profiles reconstructed from the time profiles of 50-shot accumula- 


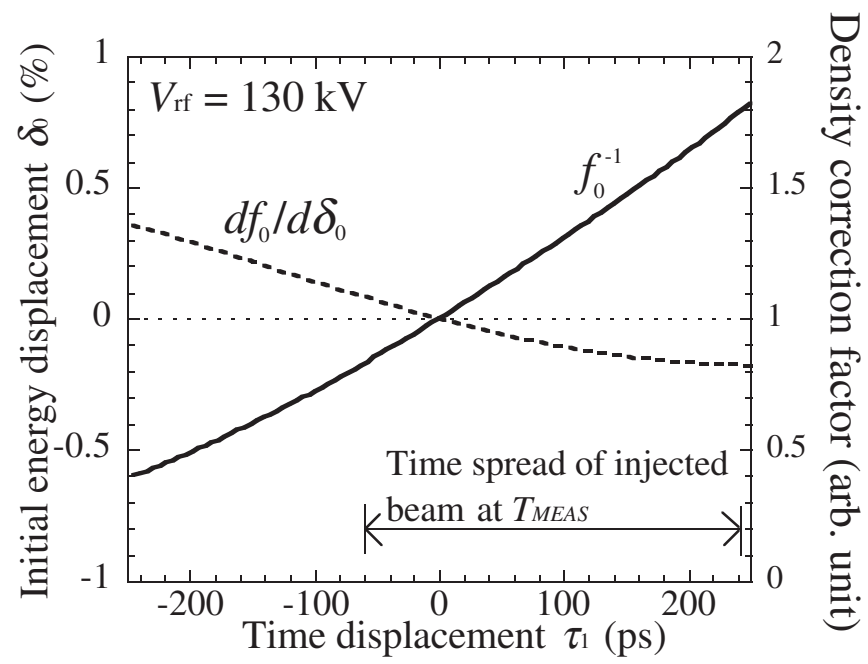

FIG. 9. Nonlinearity correction functions for the rf bucket. The solid line is the scaling function $f_{0}^{-1}$, which is the initial energy displacement $\delta_{0}$ for the calculated time displacement $\tau_{1}$. The broken line is the density correction function, which is the differential of $f_{0}$ normalized by its value at $\delta_{0}=0$.

tions and the single-shot, respectively. The static resolution was $0.026 \%$ for both profiles.

In the single-shot measurement the profile was measured using the photon counting mode, for a wide slice width along the slow-sweep axis. These conditions enhanced the brightness, but lowered the overall resolution. The worst resolution was about $0.07 \%$ (95\% width), at $\delta_{0} \approx$ $+0.45 \%$.

In the demonstrative measurements described in the previous section, the energy profiles were reconstructed from multishot profiles of the streak camera image. Multishot profiles could be used because the following known effects due to shot-by-shot fluctuations were small.

(a) Fluctuations in the bunch's center energy were $0.01 \%$ (rms), which was smaller than the static resolution.

(b) Shot-by-shot fluctuations in the injection time were less than $7.4 \mathrm{ps}$ (rms) [17] using the synchronization circuit [18]. This value was comparable to the effect of injected linac bunch length, which is discussed in Appendix B.

(c) The fast-sweep voltage phase jitter in the streak camera was smaller than 0.4 ps (rms) [19], which was much smaller than $\Delta \tau_{1}$.

\section{CONCLUSION}

This new method can be used to obtain a highresolution, bunch-by-bunch, linac electron beam energy profile. The energy profile is reconstructed from the time profile, as measured by a dual-sweep streak camera one-quarter of a synchrotron oscillation period after injection. The measured profile is sensitive to the operating parameters of the linac, and provides detailed, bunch-bybunch information on the linac beam. The method can be used in any electron linac which injects its beam into a synchrotron, by placing a streak camera at a light extraction port.

This method can be used to understand beam loss in a top-up storage ring injection process, and provides reliable information on beam loading in the linac. The method is currently being used to investigate the energy distribution of a compressed linac bunch injected into an isochronous ring.

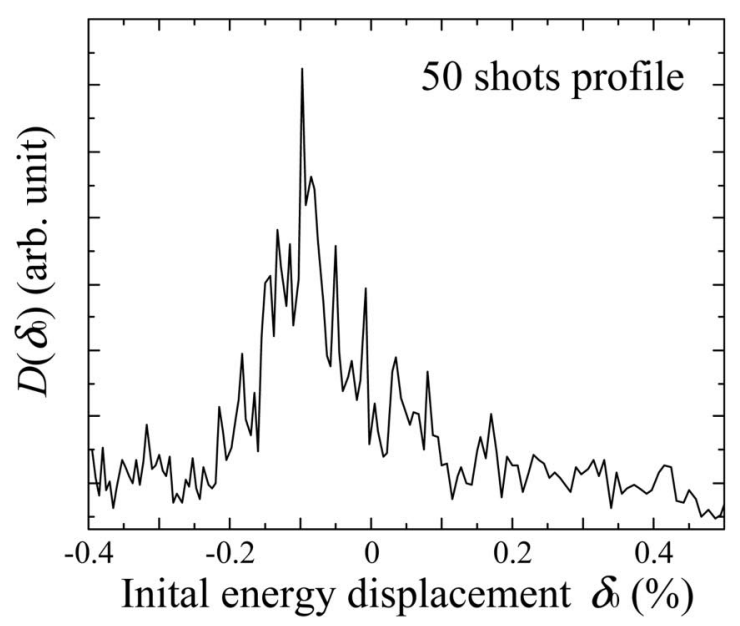

(a)

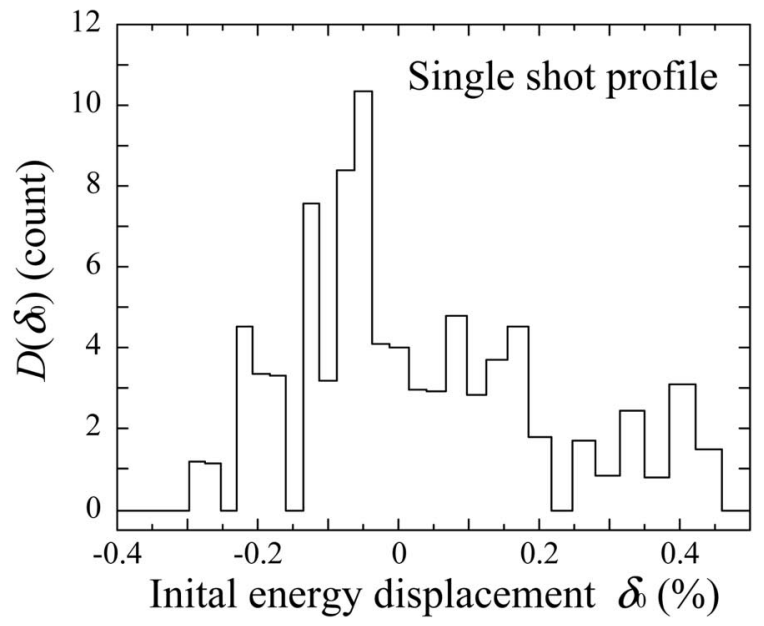

(b)

FIG. 10. Energy profiles reconstructed from (a) multishot camera images (accumulations of 50 shots) and (b) a single-shot camera image. The slice width of the oscillation image along the slow-sweep axis is $2.0 \mu \mathrm{s}$ (5 revolutions) in (a) and $8.1 \mu \mathrm{s}$ (20 revolutions) in (b). 


\section{ACKNOWLEDGMENTS}

The authors thank the linac, booster synchrotron, and storage ring staffs at SPring- 8 and NewSUBARU. The authors are especially grateful to Dr. Y. Kawashima at SPring-8 for his support, and thank Dr. Y. Miyahara for reading this article.

\section{APPENDIX A: SYNCHROTRON OSCILLATION WITH RADIATION DAMPING AND EXCITATION}

Equations (A1) and (A2) are the equations of synchrotron oscillation, including the effects of radiation damping and excitation in an electron storage ring.

$$
\begin{aligned}
d \tau= & T_{\mathrm{REV}}\left(\alpha_{1} \delta+\alpha_{2} \delta^{2}+\alpha_{3} \delta^{3}+\ldots\right) . \\
d \delta= & \frac{e V_{\mathrm{rf}} \sin \left(\phi_{S}+\omega_{\mathrm{rf}} \tau\right)-U_{0}}{E_{0}}-2 \frac{T_{\mathrm{REV}}}{\tau_{\varepsilon}} \delta \\
& +2 \sqrt{\frac{T_{\mathrm{REV}}}{\tau_{\varepsilon}}} \delta_{N} R .
\end{aligned}
$$

Here $\delta$ and $\tau$ are the relative energy displacement and time displacement of the reference particle, respectively, while $d \delta$ and $d \tau$ are the changes in these quantities per revolution. $T_{\mathrm{REV}}$ is the revolution period, $\alpha_{n}$ is the $n$th order momentum compaction factor, $V_{\mathrm{rf}}$ is the rf acceleration voltage, $\phi_{s}$ is the synchronous phase, $\omega_{\mathrm{rf}}$ is the rf angular frequency, $U_{0}$ is the radiation loss of the reference particle per revolution, $E_{0}$ is the reference energy, $\tau_{\varepsilon}$ is the longitudinal damping time, and $\delta_{N}$ is the natural energy spread. $R$ is a random Gaussian variable with unit standard deviation.

Suppose that an electron is injected at $t=0$ at the point $\left(\tau_{0}, \delta_{0}\right) . \tau_{1}$ is introduced as the value of $\tau$ at $t=T_{1 / 4}$. As $T_{1 / 4}$ is the time equal to one-quarter of the synchrotron oscillation period, $\tau_{1}$ is independent of $\tau_{0}$. A small error $\Delta t$ in $T_{1 / 4}$ is introduced to evaluate the resolution. Assuming that the oscillation amplitude is small and that $\Delta t$ is much smaller than $T_{1 / 4}, \tau_{1}$ is calculated as

$$
\begin{aligned}
\tau_{1} \approx & \exp \left(-\frac{T_{1 / 4}}{\tau_{\varepsilon}}\right)\left[-\omega_{S} \tau_{0} \Delta t+\frac{\alpha_{1}}{\omega_{S}} \delta_{0}\left(1-\frac{\Delta t}{\tau_{\varepsilon}}\right.\right. \\
& \left.\left.-\frac{\omega_{S}^{2} \Delta t^{2}}{2}\right)\right]+\frac{\alpha_{1}}{\omega_{S}} \sqrt{\frac{2 T_{1 / 4}}{\tau_{\varepsilon}}} \delta_{N} R,
\end{aligned}
$$

where $\omega_{S}$ is the synchrotron oscillation angular frequency given by

$$
\omega_{S}^{2}=-\frac{e V_{\mathrm{rf}} \omega_{\mathrm{rf}} \alpha_{1} \cos \phi_{S}}{E_{0} T_{\mathrm{REV}}} .
$$

The initial energy displacement $\delta_{0}$ is obtained from $\tau_{1}$ as

$$
\delta_{0}=\exp \left(\frac{T_{1 / 4}}{\tau_{\varepsilon}}\right) \frac{\omega_{S}}{\alpha_{1}} \tau_{1}
$$

When $\tau_{\varepsilon} \gg T_{1 / 4}$, the exponential term is near unity. In the case of NewSUBARU the damping time $\tau_{\varepsilon}$ is $12 \mathrm{~ms}$, so $\exp \left(T_{1 / 4} / \tau_{\varepsilon}\right)$ is 1.004. The intrinsic spread due to the radiation excitation $\Delta \delta_{R}$ is therefore given by

$$
\Delta \delta_{R}=\sqrt{\left(2 T_{1 / 4} / \tau_{\varepsilon}\right)} \delta_{N},
$$

which is found to be $0.0044 \%$ (standard deviation) at NewSUBARU. Thus, the contribution of $\Delta \delta_{R}$ is negligible.

The other source of error in $\delta_{0}$ is due to the finite time gate width $\Delta t$, which is referred to as $\Delta \delta_{\Delta t}$. From Eq. (A2) $\Delta \delta_{\Delta t}$ is given by

$$
\Delta \delta_{\Delta t}=\left[\frac{\omega_{S}^{2}}{\alpha_{1}} \tau_{0} \Delta t+\delta_{0}\left(\frac{\Delta t}{\tau_{\varepsilon}}+\frac{\omega_{S}^{2} \Delta t^{2}}{2}\right)\right],
$$

where $\Delta \tau_{0}$ and $\Delta \delta_{0}$ are the initial spreads of the beam. This error is dependent on the initial state, and is small provided that $\tau_{\varepsilon} \gg 1 / \omega_{S} \gg \Delta t$ and $\Delta \tau_{0} \ll\left(\alpha_{1} / \omega_{S}\right) \Delta \delta_{0}$. Assuming a linac bunch length of $20 \mathrm{ps}$, the calculated spread is $\Delta \delta_{\Delta t}=0.0042 \%+0.0027 \delta_{0}$. This contribution is also negligible.

\section{APPENDIX B: EFFECTS OF A NONLINEAR RF BUCKET}

In a nonlinear rf bucket, $\tau$ is expressed as a function of time and the initial state $\tau=f\left(\delta_{0}, \tau_{0}, t\right)$. The function $f_{0}\left(\delta_{0}\right) \equiv f\left(\delta_{0}, 0, T_{\text {MEAS }}\right)$ is used to correct for the nonlinearity. $T_{\text {MEAS }}$ is the measurement time, which is close to $T_{1 / 4}$. The initial energy $\delta_{0}$ and the profile $D\left(\delta_{0}\right)$ are obtained from $\tau_{1}$ and $T\left(\tau_{1}\right)$ by

$$
\delta_{0}=f_{0}^{-1}\left(\tau_{1}\right)
$$

and

$$
D\left(\delta_{0}\right)=\left(d f_{0} / d \delta_{0}\right) T\left(\tau_{1}\right) .
$$

The resolution of $D\left(\delta_{0}\right)$ is worse in a nonlinear rf bucket, since $T_{\text {MEAS }}=T_{1 / 4}$ is not satisfied for all $\delta_{0}$. When $T_{\text {MEAS }} \neq T_{1 / 4}$, the conditions $\partial f / \partial t \approx 0$ and $\partial f / \partial \tau_{0} \approx$ 0 are incorrect. The $\delta_{0}$-dependent errors due to $\Delta t$ and the linac bunch length $\Delta \tau_{0}$ must therefore be considered. These errors, which are referred to as $\Delta \delta_{\Delta T_{1 / 4}}$ and $\Delta \delta_{\Delta \tau_{0}}$, are given by

$$
\begin{gathered}
\Delta \delta_{\Delta T_{1 / 4}}=\frac{1}{\left(d f_{0} / d \delta_{0}\right)} \frac{\partial f}{\partial t} \Delta t, \\
\Delta \delta_{\Delta \tau_{0}}=\frac{1}{\left(d f_{0} / d \delta_{0}\right)} \frac{\partial f}{\partial \tau_{0}} \Delta \tau_{0} .
\end{gathered}
$$

The contributions $\Delta \delta_{\Delta T_{1 / 4}}$ and $\Delta \delta_{\Delta \tau_{0}}$ to the energy resolution are calculated for $V_{\mathrm{rf}}=130 \mathrm{kV}$ using the tracking 


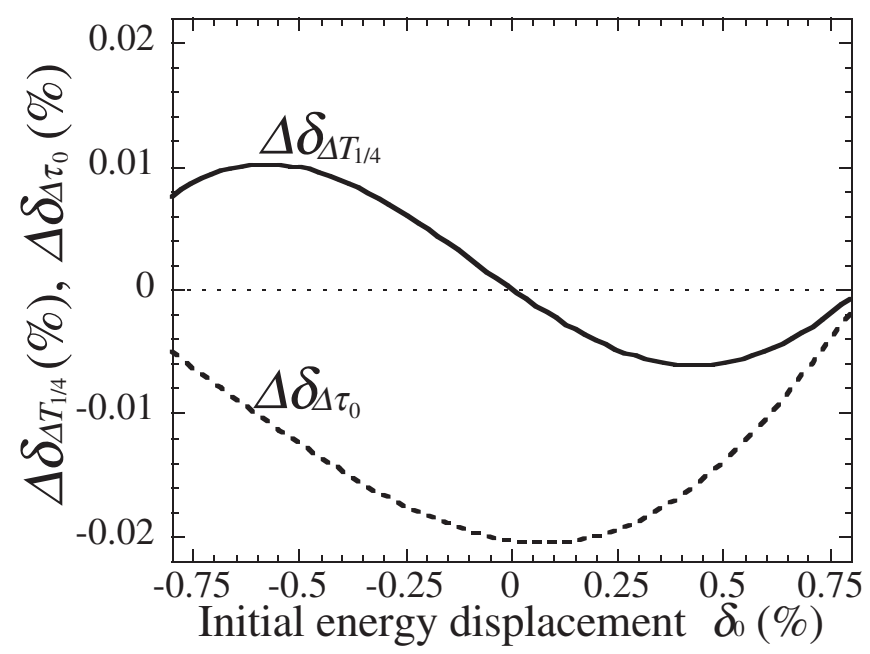

FIG. 11. Calculated contributions of the nonlinear error effects in $\delta_{0}$. The solid line shows $\Delta \delta_{\Delta T_{1 / 4}}$, the contribution due to variation of $T_{1 / 4}$, as given by Eq. (B3). The broken line shows the contribution due to the initial bunch length $\Delta \delta_{\Delta \tau_{0}}$ as given by Eq. (B4).

simulation, and displayed in Fig. 11. These contributions are smaller than the static resolution.

[1] T. Naito, H. Hayano, S. Kashiwagi, S. Takeda, and J. Urakawa, the Proceedings of the Eighteenth International Linac Conference (CERN, Geneva, Switzerland, 1996), p. 797.

[2] M. Castellano, V. Verzilov, L. Catani, A. Cianchi, G. D'Auria, M. Ferianis, and C. Rossi, in Proceedings of the 5th European Workshop on Beam Diagnostics and Instrumentation for Particle Accelerators, Grenoble, France, 2001 (ESRF, Grenoble, France, 2001), p. 46.

[3] T. Suwada, M. Satoh, and K. Furukawa, Phys. Rev. ST Accel. Beams 6, 032801 (2003).

[4] Markus Hüning, in Proceedings of the 5th European Workshop on Beam Diagnostics and Instrumentation for Particle Accelerators, Grenoble, France, 2001 (Ref. [2]), p. 56.

[5] S. Hancock, M. Lindroos, and S. Koscielniak, Phys. Rev. ST Accel. Beams 3, 124202 (2000).

[6] C. Montag, N. D'Imperio, J. Kewisch, and R. Lee, Phys. Rev. ST Accel. Beams 5, 082801 (2002).
[7] J. M. Byrd and S. De Santis, Phys. Rev. ST Accel. Beams 4, 024401 (2001).

[8] Y. Shoji, S. Hisao, T. Matsubara, T. Asaka, S. Suzuki, and Y. Kawashima, in Synchrotron Radiation Instrumentation: Eighth International Conference on Synchrotron Radiation Instrumentation , AIP Conf. Proc. No. 705 (AIP, New York, 2004), p. 53.

[9] S. Suzuki, H. Yoshikawa, T. Hori, K. Yanagida, A. Mizuno, K. Tamezane, K. Mashiko, and H. Yokomizo, in Proceedings of the Particle Accelerator Conference, Washington DC, 1993 (IEEE, Piscataway, NJ, 1993), p. 602.

[10] H. Hanaki, T. Asaka, H. Dewa, T. Kobayashi, A. Mizuno, S. Suzuki, T. Taniuchi, H. Tomizawa, and K. Yanagida, in Proceedings of the 8th European Particle Accelerator Conference, Paris, France, 2002 (EPS-IGA and CERN, Geneva, 2002), p. 1082.

[11] S. Suzuki, T. Asaka, H. Dewa, H. Hanaki, T. Kobayashi, T. Masuda, A. Mizuno, T. Taniuchi, H. Tomizawa, and K. Yanagida, in Proceedings of the Ninth European Particle Accelerator Conference, Lucerne, Switzerland, 2004 (CERN, Geneva, Switzerland, 2004), p. 1327.

[12] A. Ando, S. Amano, S. Hashimoto, H. Kinoshita, S. Miyamoto, T. Mochizuki, M. Niibe, Y. Shoji, M. Terasawa, T. Watanabe, and N. Kumagai, J. Synchrotron Radiat. 5, 342 (1998).

[13] T. Asaka, H. Hanaki, T. Kobayashi, S. Suzuki, T. Taniuchi, and K. Yanagida, in Proceedings of the Seventh European Particle Accelerator Conference, Vienna, Austria (EPS, Geneva, 2000), p. 806.

[14] T. Asaka, H. Dewa, H. Hanaki, T. Kobayashi, and A. Mizuno, in Proceedings of the 8th European Particle Accelerator Conference, Paris, France, 2002 (Ref. [10]), p. 1079.

[15] Y. Shoji, S. Hisao, and T. Matsubara, in Proceedings of the 14th Symposium on Accelerator Science and Technology (KEK, Tsukuba, 2003), p. 527, http://conference.kek.jp/ sast03/; 2P-040(S-259).

[16] Y. Shoji, Phys. Rev. ST Accel. Beams 7, 090703 (2004).

[17] H. Suzuki, H. Ego, M. Hara, T. Hori, Y. Kawashima, Y. Ohashi, T. Ohshima, N. Tani, and H. Yonehara, Nucl. Instrum. Methods Phys. Res., Sect. A 431, 294 (1999).

[18] Y. Kawashima, T. Asaka, and T. Takashima, Phys. Rev. ST Accel. Beams 4, 082001 (2001).

[19] Y. Shoji, S. Hisao, and T. Matsubara, in Proceedings of the Ninth European Particle Accelerator Conference, Lucerne, Switzerland, 2004 (Ref. [4]), p. 2353. 